\title{
GALOIS CHARACTERIZATION OF ENDOSCOPY FOR RATIONAL SIEGEL MODULAR FORMS
}

\author{
Luis V. Dieulefait
}

\begin{abstract}
We establish a relation between Galois reducibility and Endoscopy for genus 2 Siegel cusp forms which have rational eigenvalues and are unramified at 3.
\end{abstract}

\section{The theorem}

Let $f$ be a genus 2 Siegel cuspidal Hecke eigenform of weight $k>2$ and $\mathbb{Q}_{f}$ the number field generated by its eigenvalues. It is well known that if $f$ is not of Saito-Kurokawa type but it is "endoscopic" (i.e., it is in the image of the weak endoscopic lift) the compatible family of Galois representations $\left\{\rho_{f, \lambda}\right\}$ attached to $f$ (constructed by Taylor, Laumon and Weissauer for any Siegel cusp form) will be reducible over $\mathbb{Q}_{f}$, with two 2-dimensional irreducible components.

In this note we will prove that the converse statement is true, for the case $\mathbb{Q}_{f}=\mathbb{Q}$. We will have to impose a local condition at 3 and will assume that the Siegel cuspform has trivial central character. More precisely, the result is:

Theorem 1.1. Let $f$ be a genus 2 Siegel cusp form of weight $k>2$ with $\mathbb{Q}_{f}=$ $\mathbb{Q}$, such that the corresponding automorphic representation $\pi_{f}$ has multiplicity one, trivial central character, and $\pi_{f, 3}$, its local component at 3 , is unramified. Assume that for a prime $Q>4 k-5$ such that $\pi_{f, Q}$ is unramified the Galois representation $\rho_{f, Q}$ attached to $f$ reduces $($ over $\mathbb{Q}$ ) as follows:

$$
\rho_{f, Q} \simeq \sigma_{1, Q} \oplus \sigma_{2, Q}
$$

where $\sigma_{1, Q}$ and $\sigma_{2, Q}$ are 2 -dimensional irreducible representations.

Then, $f$ is endoscopic. More precisely, there exist two classical cuspidal modular forms $f_{1}, f_{2}$, of weights 2 and $2 k-2$ (respectively) such that the representation $\left\{\sigma_{1, Q} \otimes \chi^{2-k}\right\}$ is attached to $f_{1}$ and the representation $\left\{\sigma_{2, Q}\right\}$ is attached to $f_{2}$.

Remark: The irreducibility assumption of the 2-dimensional components is equivalent to assume that $f$ is not of Saito-Kurokawa type: this follows from the fact, proved by Weissauer, that if $f$ is not of Saito-Kurokawa type then the four-dimensional Galois representations $\left\{\rho_{f, \ell}\right\}$ attached to it are pure (and their determinant, $\chi^{4 k-6}$, has exponent not divisible by 4$)$.

Received by the editors October 16, 2003. Revised September 12, 2005. 


\section{Proof}

It follows from the hypothesis of multiplicity one (as proved by Weissauer) that the Galois representations attached to $f$ are symplectic. Since the compatible family $\left\{\rho_{f, \ell}\right\}$ has determinant $\chi^{4 k-6}$, we have that the two-dimensional Galois representations $\sigma_{1, Q}$ and $\sigma_{2, Q}$ are odd and have both determinant $\chi^{2 k-3}$. Also since $\pi_{f, Q}$ is unramified, it follows from a result of Faltings that the representations $\sigma_{1, Q}$ and $\sigma_{2, Q}$ are crystalline with Hodge-Tate weights $\{k-2, k-1\}$ and $\{0,2 k-3\}$, respectively.

At this point we apply the result of "existence of compatible families" in [D2] to $\sigma_{1, Q}$ (suitably twisted) and $\sigma_{2, Q}$ and thus we insert them in compatible families $\left\{\sigma_{1, \ell}\right\}$ and $\left\{\sigma_{2, \ell}\right\}$ (respectively) so that for every prime $\ell$ such that $\pi_{f, \ell}$ is unramified these two representations are also crystalline with the same HodgeTate weights that the $Q$-adic member of the corresponding family.

Comparing traces and using compatibility, we deduce from (1.1) that a similar formula holds for every $\ell$ :

$$
\rho_{f, \ell} \simeq \sigma_{1, \ell} \oplus \sigma_{2, \ell}
$$

We have to prove modularity of both of these compatible families of odd, irreducible, two-dimensional Galois representations.

Let us first show modularity of the family $\left\{\sigma_{1, \ell} \otimes \chi^{2-k}\right\}$. By assumption, $\pi_{f, 3}$ is unramified, thus $\sigma_{1,3} \otimes \chi^{2-k}$ is a Barsotti-Tate representation, irreducible, odd, with rational coefficients, and unramified outside a finite set of primes. As in Wiles original paper, using the fact that this representation has rational coefficients and results of Langlands and Tunnell, we conclude that residually this representation is either modular or reducible. Then, applying a combination of modularity liftings results of Diamond-Taylor-Wiles and Skinner-Wiles (as done in [D1] and [D2]) we conclude that $\sigma_{1,3} \otimes \chi^{2-k}$ is modular, and this gives modularity of the family $\left\{\sigma_{1, \ell} \otimes \chi^{2-k}\right\}$. The corresponding modular form $f_{1}$ must have weight 2 because for almost every $\ell$ the representations in this family are Barsotti-Tate.

This argument "à la Wiles" can not be applied to $\sigma_{2,3}$ because, even if we again have Wiles' starting point (namely, we know that residually $\bar{\sigma}_{2,3}$ is either modular or reducible), the prime 3 is too small compared with the difference $2 k-3$ of the Hodge-Tate weights to make the strategy workable.

To show modularity of the family $\left\{\sigma_{2, \ell}\right\}$ we will explode the fact that $(1.2)$ is telling us that the representations $\sigma_{2, \ell}$ can be obtained by "substracting" a modular representation from another modular representation.

A key ingredient is a result recently established by D. Whitehouse in [W], which (combined with the results of J. Arthur in [A]) proves that $\pi_{f}$ has a strong lift to an automorphic representation $\pi^{\prime}$ of $\mathrm{GL}(4, \mathbb{A})$, where $\mathbb{A}$ are the rational adeles. Thus the family $\left\{\rho_{f, \ell}\right\}$ is also attached to $\pi^{\prime}$.

We want to apply a result of Jacquet and Shalika (which appears as theorem 3.3 in [T2]), in a similar way than what is done in [T2], section 5.3.3. We have 
from (1.2) the equality of $L$-functions:

$$
L\left(\pi^{\prime}, s\right)=L\left(\sigma_{2, \ell}, s\right) L\left(f_{1} \otimes \chi^{k-2}, s\right)
$$

Observe that $\pi^{\prime}$ is the lift of $\pi_{f}$, but it is not necessarily cuspidal.

To conclude that $\sigma_{2, \ell}$ is modular, as in section 5.3.3 of [T2], we must find a prime $\ell$ such that $L\left(\sigma_{2, \ell}^{*} \otimes \sigma_{2, \ell}, s\right)$ has a simple pole at $s=1$, because in that case the result of Jacquet and Shalika implies $\sigma_{2, \ell} \simeq \sigma_{\pi_{i}, \ell}$, where $\pi_{i}$ is one of the cuspidal constituents of $\pi^{\prime}$. Then, it only remains to find a prime satisfying this condition.

Take $\ell>4 k-5$ such that the local component of $\pi^{\prime}$ (thus also of $\pi_{f}$ ) at $\ell$ is unramified. For such a prime $\ell$ the representation $\sigma_{2, \ell}$ is crystalline with Hodge-Tate weights $\{0,2 k-3\}$ and the main result of [T1] implies that there exists a totally real number field $F$ such that the restriction of $\sigma_{2, \ell}$ to the Galois group of $F$ is modular, i.e., it agrees with the Galois representation attached to a Hilbert modular form over $F$.

But, as explained in [T2], section 5.3.3, precisely from this potentially modular property (and the fact that it is preserved after solvable base change) one can deduce that $L\left(\sigma_{2, \ell}^{*} \otimes \sigma_{2, \ell}, s\right)$ does have a simple pole at $s=1$, as we wanted. This shows modularity of the family $\left\{\sigma_{2, \ell}\right\}$ and it is clear from its Hodge-Tate decomposition that it corresponds to a modular form of weight $2 k-2$. We conclude that the Siegel cusp form $f$ is endoscopic.

Final Remark: As the referee has remarked, the results of Whitehouse combined with the results of Arthur should also have consequences on multiplicity one for GSp(4) (cf. [W], page 6) and could help to remove this assumption from theorem 1.1 (these results may also imply another proof of the fact, proved by Weissauer, that the four dimensional Galois representations attached to $f$ are symplectic).

\section{References}

[A] J. Arthur, Automorphic representations of GSp(4), Contributions to automorphic forms, geometry, and number theory. Johns Hopkins Univ. Press (2004) 65-81.

[D1] L. Dieulefait, Modularity of Abelian Surfaces with Quaternionic Multiplication, Math. Res. Lett. 10 no. 2-3 (2003) 145-150.

[D2] _ Existence of families of Galois representations and new cases of the FontaineMazur conjecture, J. Reine Angew. Math. 577 (2004) 147-151.

[T1] R. Taylor, On the meromorphic continuation of degree two L-functions. Preprint 2001.

[T2] _ Galois Representations, Ann. Fac. Sci. Toulouse Math. 13 (2004) 73-119.

[W] D. Whitehouse, The twisted weighted fundamental lemma for the transfer of automorphic forms from GSp(4) to GL(4), PhD thesis, Caltech (2005).

Universitat de Barcelona, Gran Via de les Corts Catalanes, 58508007 Barcelona

E-mail address: ldieulefait@ub.edu 